\title{
Implementation of Electronic Recognition System for Railway Logistics Based on Internet of Things Technology
}

\author{
Xianwu Chu ${ }^{1, a}$, Weidong $\mathrm{Li}^{1, \mathrm{~b}}$, Zhirong Zhang ${ }^{1, \mathrm{c}}$, , Xinping Wang $^{1, \mathrm{~d}}, \mathrm{Niu} \mathrm{Li}^{1, \mathrm{e}}$ \\ ${ }^{1}$ School of Electronics and Information Engineering, DaLian JiaoTong University, DaLian 116028, \\ China \\ aemail: chuxw@djtu.edu.cn, bemail: li@djtu.edu.cn, 'email: rory_zhang@126.com, \\ demail:wxp@djtu.edu.cn, eemail: niuli@djtu.edu.cn
}

Keywords: Railway Logistics; Electronic Recognition; Information Collision; Communication Interference

\begin{abstract}
In this paper the electronic recognition system based on internet of things technology for railway logistics is designed and implemented. The 4 level information transfer mode is used between RFID Tag, train carriage identifier, station yard identifier and computer sever. Radio communication is used between RFID Tag, train carriage identifier, station yard identifier. Wired communication is used between station yard identifier and station computer. The problems of information collision between multi-tags, long-distance transmission of information and communication interference are solved in this article. The experimental results show that the system is effective when the speed of the train within $120 \mathrm{Km} / \mathrm{h}$ and it is applicable to the railway logistics.
\end{abstract}

\section{Introduction}

With the rapid development of railway logistics informationization in China, the TMIS(Transportation Management Information System),HMIS(H Management Information System) and DMIS(Dispatch Management Information System) have been used to realize real-time tracking of the train and real-time query of the goods. Data acquisition is the key problem of railway logistics informationization[1][2]. Refinement is needed in getting hold of information and real-time tracking of the goods. At present, manual process is the main method in railway logistics data acquisition. The automatic identification technology based on RFID needs to be popularized[3][4]. The technology of internet of things(IoT) should be used to realize informationization, digitization and intelligent in railway logistics and promote the transition of railroad freight transportation. The application of IoT technology will have a significant impact on the construction of railway logistics informationization[5].

In this paper the electronic recognition system based on internet of things technology for railway logistics is designed and implemented. The problems of information collision between multi-tags, long-distance transmission of information and communication interference are solved. RFID tag, identifier and communication interface unit are designed to realize railway logistics electronic recognition and meet actual requirements.

\section{System Constitution}

The electronic recognition system is shown in Figure 1.

The unique code that represents the goods' characteristics is provided by RFID tags through dedicated protocol which uses the frequency $433.2 \mathrm{MHz}$. The tag is active and its voltage is 3.3V. The code is received by train carriage identifier and the information is stored in its flash ROM and the goods state of on or off train carriage is determined. The train carriage identifier transmits information to station yard identifier through WIFI protocol which uses the frequency $2.4 \mathrm{GHz}$ and the information includes goods code, time of on or off train carriage, state of on or off train carriage, battery voltage etc. When the information reaches station yard identifier, it will be stored in identifier's flash ROM and determine the time that the train enters or leaves the station. The 
station yard identifier transmits the goods information and time to repeater or communication interface unit through CAN bus. The computer sever in railway station receives the information through communication interface unit or internet and then the information will be analyzed and processed to form documents needed. These documents can be queried by administrator in railway station[6]-[9].

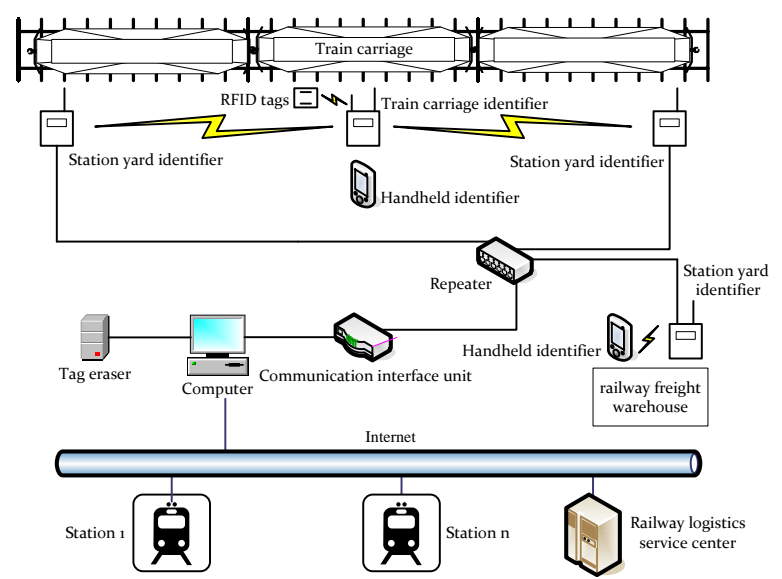

FIG. 1. Constitution of the electronic recognition system

The handheld identifier is used in train carriage and warehouse to ensure the accuracy of goods information. It can exchange information with station yard identifier through WIFI. The RFID tag erasable unit is used to rewrite information in tags, so the RFID tags can be reused.

The railway stations can exchange information through internet. The railway freight centre can know the transport conditions such as sending station, destination station, current position, estimated time of arrival etc.

\section{Electronic Recognition Mode}

Dedicated protocol and WIFI bi-directional communication technology is used in this system, and the railway logistics communication mode based on internet of things technology is realized[10]. It fundamentally changes the passive search technology of existing electronic tags and makes it possible to apply internet of things technology in railway logistics. The electronic recognition mode is shown in Figure 2.

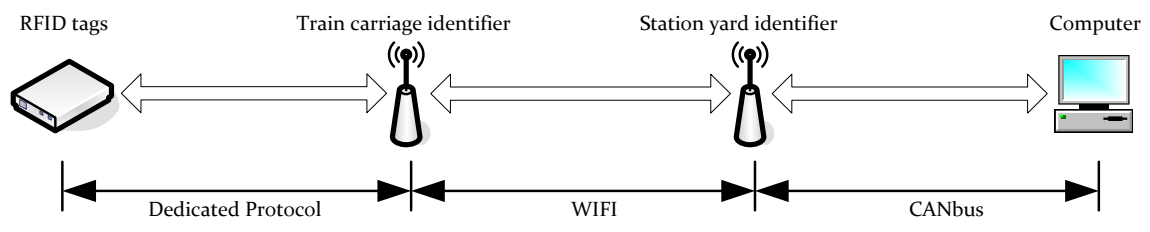

FIG. 2. Electronic recognition mode

The respective communication mode is used in this system to avoid reading miss and information collision. The train carriage identifier polling calls all of the RFID tags, only the tag that has been called can transmit information and the others can not, so the information collision can be avoided. The tags monitor radio signal continually, when one of them receives the corresponding call signal it will change into work mode otherwise it will shift between sleeping mode and monitor mode. In order to avoid information collision, when a tag gets a signal transmitted by the other tag, it will change into monitor mode again after a delay time according to algorithm[11]. When a tag gets a number for itself and there has no radio signals from other tags, it will change into transmission mode and transmits its information, at the same time, it monitor the information feedback from train carriage identifier. After the carriage identifier receives information from a tag, it will transmit a turn off signal to the corresponding tag and the tag will change into sleeping mode to avoid frequently transmission and information collision. This acknowledged mode can ensure that the information from tags has been received by train carriage 
identifier. The check code is added into the information from tags and error information will be discarded. Tags and train carriage identifier are placed in the train, so it has enough time to realize above process.

How to ensure a effective communication between train carriage identifier and station yard identifier is a key issue when the train carriage identifier passes the station yard identifier with a high speed. The train carriage searches the station yard identifier and it will link to a station yard identifier which has a high RSSI (Received Signal Strength Indicator). The information in train carriage identifier will be packed and the data package will be transmitted to station yard identifier all at once[12][13]. Simplex communication mode is used in this process to ensure a high speed data exchange.

The CAN bus communication is used between station yard identifier and station computer with a high communication speed and long distance. 32 station yard identifiers can be added on CAN bus and its baud rate is $4800 \mathrm{bps} / 4 \mathrm{Km}$. The CAN bus is divided into several segments by repeater therefore a certain station yard identifier's failure will not result in congestion collapse.

\section{Results and Conclusions}

The electronic recognition system has been tested in actual operation. The test condition is: 40 RFID tags; one train carriage identifier; one station yard identifier; the length of CAN bus is $4 \mathrm{Km}$; speed of the train is $95 \mathrm{Km} / \mathrm{h}$. The test result is shown in Figure 3.

\begin{tabular}{|c|c|c|c|c|c|c|c|c|}
\hline \multicolumn{8}{|c|}{ 鶡 Electronic Recognition System for Railway Logistics } & $-\square$ \\
\hline COM : $\operatorname{com} 1 \quad \vee$ & set time & \multicolumn{2}{|c|}{ read time } & data in & delet data & set mode & initialization & \\
\hline \multirow[t]{32}{*}{$\square 5 \mid$ CAS $\mid 192.168 .18 .6$} & SYI но. & TAG NO. & STÁTE & TCI so. & OH TINE & OFF TI & & VOLTAGE \\
\hline & & 392 & 0 & 2 & 2014-7-14 14:54:15 & 2014-7- & $14: 54: 15$ & 3. $0 \mathrm{~V}$ \\
\hline & 5 & 370 & 0 & 2 & $2014-7-14 \quad 14: 54: 18$ & 2014-7- & $14: 54: 18$ & 3. $0 \mathrm{~V}$ \\
\hline & 5 & 379 & 0 & 2 & $2014-7-14 \quad 14: 54: 18$ & $2014-7-$ & $14: 54: 18$ & 3. $0 \mathrm{~V}$ \\
\hline & 5 & 365 & 0 & 2 & $2014-7-14 \quad 14: 54: 18$ & 2014-7- & $14: 54: 18$ & 3. $0 \mathrm{~V}$ \\
\hline & 5 & 372 & 0 & 2 & $2014-7-14 \quad 14: 54: 19$ & 2014-7- & $14: 54: 19$ & 3. $0 \mathrm{~V}$ \\
\hline & 5 & 381 & 0 & 2 & $2014-7-14 \quad 14: 54: 19$ & 2014-7- & $14: 54: 19$ & 3. $0 \mathrm{~V}$ \\
\hline & 5 & 371 & 0 & 2 & $2014-7-14 \quad 14: 54: 24$ & 2014-7- & $14: 54: 24$ & 3. $0 \mathrm{~V}$ \\
\hline & 5 & 362 & 0 & 2 & $2014-7-14 \quad 14: 54: 25$ & 2014-7- & $14: 54: 25$ & 3. $0 \mathrm{~V}$ \\
\hline & 5 & 390 & 0 & 2 & $2014-7-14 \quad 14: 54: 25$ & 2014-7- & $14: 54: 25$ & $3.0 \mathrm{~V}$ \\
\hline & 5 & 375 & 0 & 2 & $2014-7-14 \quad 14: 54: 25$ & 2014-7- & $14: 54: 25$ & 3. $0 \mathrm{~V}$ \\
\hline & 5 & 394 & 0 & 2 & $2014-7-14 \quad 14: 54: 25$ & 2014-7- & $14: 54: 25$ & 3. $0 \mathrm{~V}$ \\
\hline & 5 & 383 & 0 & 2 & $2014-7-14 \quad 14: 54: 26$ & 2014-7- & $14: 54: 26$ & 3. $0 \mathrm{~V}$ \\
\hline & 5 & 369 & 0 & 2 & $2014-7-14 \quad 14: 54: 28$ & 2014-7- & $14: 54: 28$ & $3.0 \mathrm{~V}$ \\
\hline & 5 & 382 & 0 & 2 & $2014-7-14 \quad 14: 54: 13$ & 2014-7- & $14: 54: 13$ & 3. $0 \mathrm{~V}$ \\
\hline & 5 & 386 & 0 & 2 & $2014-7-14 \quad 14: 54: 13$ & 2014-7- & $14: 54: 13$ & 3. $0 \mathrm{~V}$ \\
\hline & 5 & 389 & 0 & 2 & $2014-7-14 \quad 14: 54: 13$ & $2014-7-$ & $14: 54: 13$ & 3. $0 \mathrm{~V}$ \\
\hline & 5 & 400 & 0 & 2 & $2014-7-14 \quad 14: 54: 13$ & $2014-7-$ & $14: 54: 13$ & 3. $0 \mathrm{~V}$ \\
\hline & 5 & 387 & 0 & 2 & $2014-7-14 \quad 14: 54: 13$ & $2014-7-$ & $14: 54: 13$ & 3. $0 \mathrm{~V}$ \\
\hline & 5 & 266 & 0 & 2 & $2014-7-14 \quad 14: 54: 13$ & $2014-7-$ & $14: 54: 13$ & 3. $0 \mathrm{~V}$ \\
\hline & 5 & 376 & 0 & 2 & $2014-7-14 \quad 14: 54: 13$ & $2014-7-$ & $14: 54: 13$ & 3. $0 \mathrm{~V}$ \\
\hline & 5 & 373 & 0 & 2 & $2014-7-14 \quad 14: 54: 13$ & $2014-7-$ & $14: 54: 13$ & 3. $0 \mathrm{~V}$ \\
\hline & 5 & 392 & 0 & 2 & $2014-7-14 \quad 14: 54: 15$ & $2014-7-$ & $14: 54: 15$ & 3. $0 \mathrm{~V}$ \\
\hline & 5 & 370 & 0 & 2 & $2014-7-14 \quad 14: 54: 18$ & $2014-7-$ & 14:54:18 & 3. $0 \mathrm{~V}$ \\
\hline & 5 & 379 & 0 & 2 & $2014-7-14 \quad 14: 54: 18$ & $2014-7-$ & 14:54:18 & 3. $0 \mathrm{~V}$ \\
\hline & 5 & 365 & 0 & 2 & $2014-7-14 \quad 14: 54: 18$ & $2014-7-$ & $14: 54: 18$ & 3. $0 \mathrm{~V}$ \\
\hline & 5 & 372 & 0 & 2 & $2014-7-14 \quad 14: 54: 19$ & $2014-7-$ & $14: 54: 19$ & 3. $0 \mathrm{~V}$ \\
\hline & 5 & 381 & 0 & 2 & $2014-7-14 \quad 14: 54: 19$ & $2014-7-$ & $14: 54: 19$ & 3. $0 \mathrm{~V}$ \\
\hline & 5 & 371 & 0 & 2 & $2014-7-14 \quad 14: 54: 24$ & $2014-7-$ & $14: 54: 24$ & 3. $0 \mathrm{~V}$ \\
\hline & 5 & 362 & 0 & 2 & $2014-7-14 \quad 14: 54: 25$ & $2014-7-$ & $14: 54: 25$ & 3. $0 \mathrm{~V}$ \\
\hline & 5 & 390 & 0 & 2 & $2014-7-14 \quad 14: 54: 25$ & $2014-7-$ & $14: 54: 25$ & 3. $0 \mathrm{~V}$ \\
\hline & 5 & 375 & 0 & 2 & $2014-7-14 \quad 14: 54: 25$ & $2014-7-$ & $14: 54: 25$ & $3.0 \mathrm{~V}$ \\
\hline DELET & 5 & 394 & 0 & 2 & $2014-7-14 \quad 14: 54: 25$ & 2014-7- & $14: 54: 25$ & 3. $0 \mathrm{~V}$ \\
\hline & 5 & 383 & 0 & 2 & $2014-7-14 \quad 14: 54: 26$ & 2014-7- & $+14: 54: 26$ & 3. $0 \mathrm{~V}$ \\
\hline & 5 & 369 & 0 & 2 & $2014-7-14 \quad 14: 54: 28$ & 2014-7- & $14: 54: 28$ & $3.0 \mathrm{~V}$ \\
\hline
\end{tabular}

FIG. 3. Test Result

As shown in Fig. 3, train carriage identifier's serial number is 2, station yard identifier's serial number is 5; RFID tags' serial number is 361-400. State 0 indicates that the tag is on train, and the on train time is same with the off train time in this situation. State 1 indicates that the tag is off train,and the off train time is corresponding time. The voltage of tags' battery is also in the figure and if the voltage is less than $2.3 \mathrm{~V}$ the tag will generate a alarm signal. The 40 RFID tags' information is received by computer in 2 seconds.

The application of electronic recognition based on internet of things technology for railway logistics is researched in this paper. Credible and long distance communication is realized. The electronic recognition system has characteristics such as low error rate, low misreading rate, low collision rate and it is credible. 


\section{Acknowledgement}

This work was financially supported by the National Natural Science Foundation of China (61471080).

\section{References}

[1] Zhou Qinian, Chen Lingling, Li Ge, Zhang Zhenhao. Research on internet of things technology. Lecture Notes in Electrical Engineering, v 107 LNEE, p 253-260, 2012, Computer, Informatics, Cybernetics and Applications - Proceedings of the CICA 2011.

[2] Shi Guo-liang, Liu Yu-xi. Research on key technologies for Internet of things. Applied Mechanics and Materials, v 190-191, p 308-312, 2012.

[3] Guo Zhida, Zhang ZhiRong, Li WeiDong. Establishment of intelligent identification management platform in railway logistics system by means of the Internet of Things. Procedia Engineering, v 29, p 726-730, 2012.

[4] Wang HaiQun, Xue WenJun. Development and application of the railway transportation management information system. Applied Mechanics and Materials, v 385-386, p 1848-1851, 2013.

[5] Deng Yang, Ou Zhonghong; Komu Miika, Looga Vilen, Yla-Jaaski Antti. Anatomy of the connecting architecture of Internet of Things. Proceedings - 2012 IEEE 2nd International Conference on Cloud Computing and Intelligence Systems, IEEE CCIS 2012, v 3, p 1230-1234, November 13, 2013.

[6] Wu Miao, Lu Ting-Jie, Ling Fei-Yang, Sun Jing, Du Hui-Ying. Research on the architecture of Internet of Things. ICACTE 2010 - 2010 3rd International Conference on Advanced Computer Theory and Engineering, Proceedings, v 5, p V5484-V5487, 2010.

[7] Sun Fu-Zhao. Research on Internet of Things data exchange based on SOA. Lecture Notes in Electrical Engineering, v 273 LNEE, n VOL. 4, p 221-227, 2014, Proceedings of the 9th International Symposium on Linear Drives for Industry Applications, LDIA 2013.

[8] Shu Qichong, Zhong, Shuang, Zeng, Xiangyu. The architecture of the internet of things in railway logistics. ICLEM 2012: Logistics for Sustained Economic Development - Technology and Management for Efficiency - Proceedings of the 2012 International Conference of Logistics Engineering and Management, p 1326-1332, 2012.

[9] Sun Youwang, Zhu Ye, Li Yunqing. Relevant problem of modern logistics developments in railway. Proceedings of the Conference on Traffic and Transportation Studies, ICTTS, p 1529-1533, 2002.

[10] Che Xiaoping, Maag Stephane. A passive testing approach for protocols in internet of things. Proceedings - 2013 IEEE International Conference on Green Computing and Communications and IEEE Internet of Things and IEEE Cyber, Physical and Social Computing, GreenCom-iThings-CPSCom 2013, p 678-684, 2013.

[11] Kalmar Andras, Vida Rolland, Maliosz Markosz. Context-aware addressing in the Internet of Things using Bloom filters. 4th IEEE International Conference on Cognitive Infocommunications, CogInfoCom 2013 - Proceedings, p 487-492, 2013.

[12] Lei Xiang, Wang Xi Fu, Li Li. Railway information integration and sharing mode researching based on internet of things. Advanced Materials Research, v 694 697, p 3353-3356, 2013.

[13] Chen Luyu, Ren Guangwei. The research of data mining technology of privacy preserving in sharing platform of internet of things. Communications in Computer and Information Science, $\mathrm{v}$ 312 CCIS, p 481-485, 2012. 